\title{
Características psicosociales en niños en contextos de riesgo y de no riesgo
}

\author{
Ivonne González Arratia L. F. \\ José Luis Valdez Medina \\ Alejandra Domínguez Espinosa \\ Joaquina Palomar Lever \\ Sergio González Escobar
}

Diversas investigaciones han puesto en evidencia la importancia de la familia para el desarrollo óptimo del individuo, así como los efectos debido a su ausencia. Por ello, se investigó si existían diferencias en niños que viven en familia y niños huérfanos, en variables psicológicas tales como: Autoestima, Locus de control, Enfrentamiento a los problemas de la vida, Funciones del Yo y Relaciones intrafamiliares, para lo cual se trabajó con una muestra compuesta por 355 niños de ambos sexos que viven en familia (200) y en situación de orfandad (155) entre 9 y 14 años de edad de la ciudad de Toluca, México. Los resultados indican diferencias en cada una de las escalas aplicadas a favor de los niños de familia.

características psicológicas / factores de riesgo / protección

Psychosocial characteristics in children at-risk and non-risk environments

Some researchers have put in evidence the importance of the family for the individual optimal development, as well as the effects due to the lack of this. Reason why it was investigated if there are some differences between children who live in family and children who live in orphaned situation, in psychological variables such as: self-esteem, locus of control, intra-family relationships, confrontation to the problems in life, and functions of the I (self). It worked with a sample composed by 355 children of both sexes that live in family (200) and (155) who live in orphaned situation. All the children are between 9 to 14 years old, and live in Toluca, Mexico State City. The results indicate differences in each one of the scales applied in favor of the family children.

psychological characteristics / risk factors / protection 
El presente estudio parte de la consideración de que si el desarrollo del niño transcurre en una situación o ambiente considerado de riesgo (como el hecho de estar fuera del núcleo familiar -un albergue-, o bien que en la comunidad existan factores sociales de riesgo -alcoholismo, drogadicción-, posiblemente existan diferencias en las características de personalidad (Autoestima, Locus de control, Enfrentamiento a los problemas, Funciones del Yo y Relaciones intrafamiliares) respecto de cuando no hay estas condiciones en la vida del niño. El objetivo de la presente investigación es identificar las diferencias o similitudes de las características psicológicas de niños que viven en contextos considerados de no riesgo (dentro de una familia) y de niños sin la presencia de esta, o contextos de riesgo, dado que se encuentran en albergues por motivo de orfandad.

El papel de la familia ha sido crucial en el desarrollo y la socialización del individuo. Así, los teóricos del desarrollo destacan que es la red primaria de apoyo en donde se establecen relaciones afectivas, así como la identificación de figuras significativas para el pequeño. Es considerada también como el sistema fundamental en donde tienen lugar experiencias que influirán a lo largo de la vida, especialmente del niño pequeño, independientemente de su configuración y su dinámica.

Pero la familia también ha sido referida como factor de riesgo, debido a que los niños tienen que superar circunstancias adversas encontradas en su familia de origen y que repercute en diversos ámbitos: físico, psicológico, emocional y social (Loredo, 1994; Secadas, 1984; Córdova, 2006; Rutter, 1985, en Kotliarenco, Cáceres \& Fontecilla, 1996).

Una buena parte de niños y adolescentes son víctimas de violencia doméstica, abuso sexual, negligencia y abandono. Al respecto, las investigaciones refieren que el rechazo y la carencia de amor por parte de los padres o de una persona significativa para el niño, se relaciona con una baja autoestima, sentimientos de incompetencia, hostilidad, disminución de la confianza en sí mismos y presencia de conductas antisociales en los pequeños (Maher, 1990 en Martínez, 2006).

Ante circunstancias como el abandono por parte de la familia o la ausencia definitiva de esta, las instituciones tanto gubernamentales como no gubernamentales, religiosas y de asistencia privada, son importantes fuentes de apoyo y protección para los niños y adolescentes y se convierten en la forma clásica de beneficencia, que tienen como funciones primordiales la de cultivar la personalidad del pequeño, prepararlo para su misión trascendente y suplir en lo posible la carencia de hogar (Martínez, 2006).

Específicamente en el caso del Estado de México, actualmente las políticas asistenciales son operadas por el 
llamado Sistema Integral de la Familia del Estado de México (Difem), y están dirigidas a la protección de grupos vulnerables de bebés, niños y adolescentes en situación de desamparo, en albergues y casas cuna (Guadarrama, 2001). Las funciones de los servicios asistenciales (Villa Hogar, Villa juvenil, Albergue Temporal Infantil), son el dar casa-habitación, alimento y vestido a cada niño y joven ingresado, proporcionar el ingreso a una institución educativa de acuerdo al nivel y grado académico que le corresponda para continuar su desarrollo, ofrecer diferentes opciones de capacitación técnica como artesanal para un trabajo productivo y brindar atención médica y psicológica. Para ello, cuentan con recursos tanto personales (psicólogos, trabajadores, sociales, personal administrativo, etcétera), como financieros, que le permiten a estas instituciones operar de manera adecuada.

Sin embargo, no todas las instituciones de asistencia cuentan con la misma infraestructura (recursos humanos, materiales y financieros) ni con los ingresos para el sostenimiento adecuado de los niños y adolescentes. Así, existen también instituciones con menores recursos cuyo objetivo es dar atención básica (alimentación, vestido), que tienen que enfrentar dificultades para la manutención y, por lo tanto, su apoyo a otras actividades (actividades extraescolares, atención médica, diversión, entre otras) es restringido.
Además, le falta capacitación al personal que tiene a su cargo la atención de los menores, quienes han sido abandonados o han sufrido conflictos familiares, separación de los padres, muerte de uno o de ambos padres, abuso, maltrato, consumo de drogas o alcohol, violencia psicológica o física, psicopatología en la familia, pobreza, entre otros.

Hemos constatado, entonces, que existen diferencias entre las instituciones a pesar de que tienen la misma intención de dar protección a los grupos vulnerables. Estas diferencias también hacen suponer que el tipo de interacción y relación que establecen entre sí también sean distintas. Así, en los reportes de Lucas y Koller (2007, citados en Munist, Suárez, Krauskopf y Silber, 2007), mencionan que no es posible afirmar si una institución es o no un factor de riesgo o protección. Lo ideal es que se busque identificar en cada entidad aquellos elementos favorables y aquellos que no lo son para el buen desarrollo de los niños y adolescentes atendidos.

Los estudios realizados no han examinado suficientemente el contexto familiar y los albergues; por ello, es necesario seguir investigando variables psicológicas, considerando estos contextos de riesgo y no riesgo. Para esto se tomó como base el enfoque de riesgo, puesto que su conocimiento nos permite anticipar una atención adecuada, oportuna y realizar acciones para prevenir o reducir la aparición del hecho indeseado. 
Pero, además, por lo que menciona Perry y Montiel (1996, citado en Sandoval, 2006), de que hay que concebir el riesgo en el contexto de sus consecuencias para la vida de los individuos, y porque puede, incluso, interrumpir instantáneamente el curso normal de sus vidas.

Se puede entender como factor de riesgo cualquier característica o cualidad de una persona o comunidad que se sabe va unida a una elevada probabilidad de dañar la salud. También son variables que pueden afectar negativamente el desarrollo de las personas. Asimismo, cuando se habla de factores de riesgo se hace referencia a las situaciones contextuales o personales que, al estar presentes, incrementan la probabilidad de desarrollar problemas emocionales, conductuales o de salud, y que dañan, impiden o limitan el potencial de un individuo (Jessor, 1993; Rodríguez, 1995; Rojas, 2007; Rutter, 1985, 1987).

Por otro lado, están los factores protectores, que son las condiciones que impiden la aparición del riesgo, de las conductas conducentes a él; esto reduce la vulnerabilidad y favorece la resistencia al daño; es decir que son las condiciones o los entornos capaces de favorecer el desarrollo de individuos o grupos, $\mathrm{y}$, en muchos casos, de reducir los efectos de circunstancias desfavorables.

Se puede distinguir entre factores externos e internos. Los externos se refieren a condiciones que actúan reduciendo la probabilidad de daños; por ejemplo, la familia, que actúa como factor protector cuando esta proporciona seguridad y afecto al pequeño, lo que propicia el desarrollo de confianza en sí mismo (Bromley, 1969). Por otro lado, los internos son los atributos de la propia persona: personalidad, autoestima, asertividad, entre otros.

En este sentido, es necesario destacar que existen importantes elementos psicológicos que aumentan el potencial protector y contribuyen a la mediación del riesgo, y que se construyen a partir del establecimiento de la autoestima (Verduzco, 2004; González Arratia, 2007), de las posibilidades de probar las destrezas para enfrentar los problemas (Enfrentamiento) (Góngora, 2000; Góngora \& Reyes Lagunes, 2000) y asumir las responsabilidades frente a ellos (Locus de control) (Andrade, 1984), de la percepción de adecuadas relaciones familiares (Relaciones intrafamiliares) (Rivera, 1999; Córdova, 2006) y de la capacidad de adaptación (Funciones del Yo) (Bellack, 1994).

Rojas (2007) refiere que se producen una serie de transacciones que dan lugar a procesos que disminuyen la posibilidad del daño. En el caso de los niños con déficits de estas características, favorecen e incrementan el impacto de los factores de riesgo, dado que estas particularidades psicológicas disminuyen o aumentan el impacto del riesgo que da lugar a las fortalezas y debilidades, cuyo balance se expresa en la mayor o menor resistencia a la adversidad. 
De esta manera, a pesar de las múltiples investigaciones en las que se toman en cuenta las diferencias entre los niños de familia y los niños de albergue, se requiere continuar explorando el funcionamiento psicológico; por ello, el estudio de estas diferencias se va a centrar en factores de riesgo y de protección, a fin de que se puedan contrarrestar los posibles efectos de los factores de riesgo.

Esto es así porque se deben identificar con cierta periodicidad tanto factores de riesgo como de protección, debido a que la vulnerabilidad es un estado en permanente cambio (Donas, 1999).

Por lo tanto, se tiene que considerar que el hecho de permanecer en una familia puede no ser suficiente factor protector que permita al niño desenvolverse mejor debido a las interacciones en el interior de esta, y al mismo tiempo no todas las instituciones cuentan con los suficientes recursos para proporcionar la menor atención y seguridad. Así, se hace necesario identificar si el ambiente donde se desarrolla el niño es un factor importante para producir diferencias psicológicas en estos grupos.

\section{MÉTODO}

\section{Participantes}

Se utilizó un muestreo no probabilístico de tipo intencional, compuesto por 355 niños de escuelas y albergues públicos del área metropolitana y conurbada de la ciudad de Toluca, Estado de México. Hombres y mujeres con un rango de edad de 9 a 14 años ( $\overline{\mathrm{x}}=11.36$ años $)$, que se dividió en cuatro grupos de acuerdo con los siguientes criterios:

\section{Grupo 1}

Primero se seleccionaron dos escuelas, una considerada de alto riesgo y la otra de bajo riesgo. Los criterios para elegir la primera de estas fueron los recogidos después de una entrevista con la directora, quien expresó como problemáticas del entorno escolar las siguientes: presencia de pandillas alrededor de la escuela, venta de drogas al menudeo, alcoholismo en algún miembro de la familia, consumo de drogas por parte de los padres, violencia intrafamiliar, abuso y maltrato a los niños por parte de los padres. A este grupo se le denominó escuela de alto riesgo, y para facilitar su presentación se le identificará con las siglas EAR.

\section{Grupo 2}

La escuela considerada como de no riesgo (EBR) cuenta con todos los servicios públicos y la directora no reportó la presencia de pandillas, drogadicción, alcoholismo, violencia ni maltrato físico a los niños.

\section{Grupo 3}

En el caso de los participantes de instituciones de asistencia social se eligie- 
ron los albergues que pertenecen al sistema DIF (Estado de México), que fueron considerados como de bajo riesgo (ABR). Estos se seleccionaron por sus características de contar con presupuesto estatal para su manutención; cuentan con un cuidador por cada seis niños ubicados en pequeñas casas; asisten a una escuela pública; y cuentan con todos los servicios, actividades extraescolares, deportivas y recreativas; asimismo, cuentan con grupos de apoyo psicológico y pedagógico. De acuerdo con sus objetivos y programas la institución considera que representa un ambiente de no riesgo en este escenario de apoyo familiar y asistencia a grupos vulnerables.

\section{Grupo 4}

Para el grupo de albergues de alto riesgo (AAR) se seleccionaron albergues de asistencia privada, con la característica de ser una casa bajo el cuidado de dos o tres personas, con 10 a 20 niños y niñas.

Cuentan con pocos recursos económicos para su manutención, pues dependen de beneficios que les proporciona la sociedad civil. Por esta situación, no es posible el desarrollo de actividades extraescolares ni deportivas; asisten a diferentes escuelas, algunos niños en la mañana y otros en la tarde. Además, existe la posibilidad de hacinamiento, dado que los espacios son reducidos. En la zona donde se encuentra existen pandillas, y, según las cifras del municipio, es una zona de altos índices de robo y violencia.

A fin de facilitar las características de la muestra, se presenta la siguiente tabla:

\section{Tabla 1}

Características de los participantes

\begin{tabular}{|c|c|c|c|c|}
\hline & \multicolumn{2}{|c|}{$\begin{array}{c}\text { Viven en familia } N=200 \\
\text { Bajo riesgo } N=188\end{array}$} & \multicolumn{2}{|c|}{$\begin{array}{l}\text { Viven en albergue } N=155 \\
\text { Alto riesgo } N=167\end{array}$} \\
\hline & $\begin{array}{c}\text { Escuela de } \\
\text { alto riesgo } \\
\text { (EAR) } \\
N=100\end{array}$ & $\begin{array}{c}\text { Escuela de } \\
\text { bajo riesgo } \\
\quad(E B R) \\
N=100\end{array}$ & $\begin{array}{l}\text { Albergue de } \\
\text { alto riesgo } \\
\qquad(A A R) \\
N=67\end{array}$ & $\begin{array}{c}\text { Albergue de } \\
\text { bajo riesgo } \\
(A B R) \\
N=88\end{array}$ \\
\hline \multicolumn{5}{|l|}{ Niño } \\
\hline$N=189$ & 59 & 42 & 35 & 53 \\
\hline \multicolumn{5}{|l|}{ Niña } \\
\hline$N=166$ & 41 & 58 & 32 & 35 \\
\hline
\end{tabular}




\section{Instrumentos}

- Prueba multidimensional y multisituacional de enfrentamiento a los problemas (Góngora y Reyes, 2000).- Mide el Enfrentamiento como rasgo con reactivos que se inician con la pregunta: Cuando tengo problemas en la vida yo (situación general) y para medir el enfrentamiento como estado (con mi familia, salud, amigos y escuela). Escala tipo likert pictórica con siete opciones de respuesta, con 18 reactivos cada una y seis factores: directorevalorativo, emocional-negativo, emocional-evasivo, directo, revalorativo y social-emocional negativo. Góngora (2000) explica que se alcanza un coeficiente Alpha de Cronbach .7506 entre las situaciones.

- Locus de control.- Contiene 30 reactivos con dos opciones de respuesta (Sí-No) divididos en tres factores: instrumentalidad, afectividad $\mathrm{y}$ fatalismo con un Alpha $=0.73$ (Andrade, 1984).

- Autoestima.- Cuenta con 32 reactivos con dos opciones de respuesta (SíNo) y los factores son: devaluación a nivel social, cumplir con expectativas sociales, inseguridad, familia, aspectos negativos de la autoestima y escuela, que explican el $42,53 \%$ de la varianza y un Alpha de Cronbach de .8300 (Verduzco, 2004).
- Funciones del Yo.- Se aplicó el cuestionario de Evaluación de las Funciones del Yo (EFY), exclusivamente el área o la dimensión de dominio-competencia desarrollado por Bellak (1994); consta de 10 reactivos con cuatro opciones de respuesta. En la investigación se obtuvo un Alpha = .666.

- Relaciones intrafamiliares: Se aplicó la versión corta de Rivera (1999), que consta de 37 reactivos con cinco opciones de respuesta y tres factores: expresión, dificultades y unión, y con una confiabilidad a través de Alpha de Cronbach de 9149 (Córdova, 2005).

\section{Procedimiento}

Una vez seleccionados los escenarios y los participantes, se procedió a la aplicación de cada una de las escalas, dentro del horario laboral de las escuelas y los albergues. Las aplicaciones se realizaron de manera grupal en dos sesiones, la primera aplicación fue de 20 minutos y la segunda de 40 minutos, aclarándose las dudas que surgieron en el momento. Todos los participantes accedieron voluntariamente al estudio.

\section{RESULTADOS}

Con el objetivo de conocer si existían diferencias entre las variables Relaciones intrafamiliares, Locus de control, 
Autoestima, Funciones del Yo y Enfrentamiento de acuerdo con la condición de vida (viven dentro de la familia y albergue) se utilizó una t de Student, y para la variable grupo y grupo (escuela de alto y bajo riesgo y albergue de alto y bajo riesgo), se usó un análisis de varianza, incluyéndose una prueba poshoc de Scheffé, con un nivel de significación estadística de $\mathrm{p}=.05 \mathrm{o}$ menor.

Para la variable Condición de vida, utilizando la prueba $t$ de Student, se encontraron diferencias estadísticamente significativas en las dimensiones de la escala de autoestima, que indican que los niños que viven en el albergue muestran mayor devaluación, $\mathrm{t}=-5.61$, $\mathrm{p}=.00$, media $.42(.24)$; requieren de cumplir expectativas sociales, $\mathrm{t}=-4.72, \mathrm{p}=.00$, media .36(.36) más inseguridad $\mathrm{t}=-5.81, \mathrm{p}=.00$, media .43(.27) y más aspectos negativos de la autoestima, $\mathrm{t}=-5.33, \mathrm{p}=.00$, media .38(.26) en contraste con los niños que viven en familia, que muestran más aspectos positivos dentro de su familia y la escuela $\mathrm{t}=.51, \mathrm{p}=.00$, media .31(.19).

Para la escala de Locus de control y Condición de vida, se encontró que los niños que viven en albergue muestran mayor locus de control externo, $\mathrm{t}=-7.66, \mathrm{p}=.00$, media $.43(.23)$ mientras que los niños que viven en familia, muestran mayor locus de control interno: $\mathrm{t}=3.29, \mathrm{p}=.00$, media .68(.19).
En el caso del instrumento de las Funciones del Yo se encontró que los niños que viven en albergues muestran mayor dominio competencia $\mathrm{t}=-2.65$, $\mathrm{p}=.00$, media $1.89(.52)$.

En la variable Enfrentamiento a los problemas también se encontraron diferencias a través de la prueba $t$ de Student en algunas dimensiones de la escala, que indican que los niños que viven en albergues muestran mayor enfrentamiento de tipo emocional $\mathrm{t}=-4.41, \mathrm{p}=.00$, media $.4 .75(1.27) \mathrm{y}$ evasivo 4.73(1.24) en los problemas de la vida.

Para los problemas familiares presentan mayor enfrentamiento emocional $\mathrm{t}=-3.17, \mathrm{p}=.00$, media $4.94(1.23)$ y evasivo, 4.29 (1.35). En el caso de la dimensión de los problemas de la escuela, se obtuvo un enfrentamiento con estilo negativo $\mathrm{t}=-3.19, \mathrm{p}=.00$, media 4.96(1.34) y evasivo con una media de 4.89(1.29), y, por último, para los problemas con los amigos indican tener mayor enfrentamiento de tipo evasivo $t$ $=-3.89, \mathrm{p}=.00$ y media $4.89(1.29)$, en comparación con los niños que viven en familia.

Siguiendo con la misma variable de comparación la Condición de vida (viven en familia/viven en albergue) y la escala de Relaciones intrafamiliares, se encontró que es mayor en los niños que viven en familia la dimensión expre- 
sión, $\mathrm{t}=4.88, \mathrm{p}=.00$, media $4.27(.59)$ $\mathrm{y}$ unión $\mathrm{t}=6.02 \mathrm{p}=.00$, media 4.54(.57), mientras que los niños del albergue perciben mayores dificultades $\mathrm{t}=7.16, \mathrm{p}=.00$, media 2.67(.75).

En cuanto a las diferencias por grupo se usó un análisis de varianza incluyéndose una prueba poshoc para cada una de las escalas aplicadas. Así, en la escala de Relaciones Intrafamiliares, en la dimensión Expresión, se encontró una diferencia significativa entre el grupo de escuela de bajo riesgo respecto de los albergues; mayor dificultad en el albergue de alto riesgo y mayor unión en la escuela de bajo riesgo respecto de los albergues (véase tabla 2). En el caso de la variable Autoestima y grupo, también se encontraron diferencias estadísticamente significativas que indican mayor devaluación social e inseguridad, y tienen más aspectos negativos en su autoestima los niños del grupo de AAR mientras que los niños del grupo del ABR consideran que deben cumplir con expectativas sociales y los del EBR tienen en cuenta aspectos de la familia como parte de su autoestima; en la dimensión escuela no se encontraron diferencias significativas (véase tabla 3 ).

Tabla 2

Análisis de varianza Relaciones intrafamiliares Diferencias por dimensión y grupo

\begin{tabular}{|c|c|c|c|c|c|c|}
\hline Dimensión & Grupos & Media & $\begin{array}{c}\text { Diferencias } \\
\text { entre los grupos }\end{array}$ & $f$ & gl & $\mathbf{P}$ \\
\hline Expresión & $\begin{array}{l}1=\text { esc. alto riesgo } \\
2=\text { esc. bajo riesgo } \\
3=\text { alb. bajo riesgo } \\
4=\text { alb. alto riesgo }\end{array}$ & $\begin{array}{l}4.23 \\
4.32 \\
3.90 \\
3.98\end{array}$ & $\begin{array}{l}1,3 \\
2,4 \\
1,4\end{array}$ & 8.76 & 354 & .000 \\
\hline Dificultades & $\begin{array}{l}1=\text { esc. alto riesgo } \\
2=\text { esc. bajo riesgo } \\
3=\text { alb. bajo riesgo } \\
4=\text { alb. alto riesgo }\end{array}$ & $\begin{array}{l}3.20 \\
3.35 \\
2.81 \\
2.49\end{array}$ & $\begin{array}{l}1,4 \\
2.4\end{array}$ & 19.65 & & .000 \\
\hline Unión & $\begin{array}{l}1=\text { esc. alto riesgo } \\
2=\text { esc. bajo riesgo } \\
3=\text { alb. bajo riesgo } \\
4=\text { alb. alto riesgo }\end{array}$ & $\begin{array}{l}4.45 \\
4.62 \\
4.08 \\
4.13\end{array}$ & $\begin{array}{c}1,3,4 \\
2,4\end{array}$ & 14.10 & & .000 \\
\hline
\end{tabular}


Tabla 3

Análisis de varianza Autoestima

Diferencias por dimensión y grupo

\begin{tabular}{|c|c|c|c|c|c|c|}
\hline Dimensión & Grupos & Media & $\begin{array}{c}\text { Diferencias } \\
\text { entre los grupos }\end{array}$ & f & gl & $\mathbf{P}$ \\
\hline \multirow{4}{*}{$\begin{array}{l}\text { Devaluación } \\
\text { social }\end{array}$} & $1=$ esc. alto riesgo & 3.01 & 1,3 & 14.6 & 354 & .000 \\
\hline & $2=$ esc. bajo riesgo & 2.12 & 2,4 & & & \\
\hline & $3=$ alb. bajo riesgo & 3.73 & & & & \\
\hline & $4=$ alb. alto riesgo & 3.86 & & & & \\
\hline \multirow{4}{*}{$\begin{array}{l}\text { Cumplir con } \\
\text { expectativas } \\
\text { sociales }\end{array}$} & $1=$ esc. alto riesgo & .66 & 1,3 & 9.12 & & .000 \\
\hline & $2=$ esc. bajo riesgo & .53 & 2,3 & & & \\
\hline & $3=$ alb. bajo riesgo & 1.21 & 3,4 & & & \\
\hline & $4=$ alb. alto riesgo & .95 & & & & \\
\hline \multirow[t]{4}{*}{ Inseguridad } & $1=$ esc. alto riesgo & 2.04 & 1,4 & 16.6 & & .000 \\
\hline & $2=$ esc. bajo riesgo & 1.28 & 2,3 & & & \\
\hline & $3=$ alb. bajo riesgo & 2.48 & & & & \\
\hline & 4= alb. alto riesgo & 2.83 & & & & \\
\hline \multirow[t]{4}{*}{ Familia } & $1=$ esc. alto riesgo & 3.79 & 1,3 & 23.2 & & .000 \\
\hline & $2=$ esc. bajo riesgo & 3.81 & 2,4 & & & \\
\hline & $3=$ alb. bajo riesgo & 3.01 & & & & \\
\hline & 4= alb. alto riesgo & 3.14 & & & & \\
\hline \multirow{4}{*}{$\begin{array}{l}\text { Aspectos } \\
\text { negativos de } \\
\text { la autoestima }\end{array}$} & $1=$ esc. alto riesgo & 1.75 & & 12.6 & & .000 \\
\hline & $2=$ esc. bajo riesgo & 1.20 & $2,3,4$ & & & \\
\hline & $3=$ alb. bajo riesgo & 2.29 & & & & \\
\hline & $4=$ alb. alto riesgo & 2.32 & & & & \\
\hline \multirow[t]{4}{*}{ Escuela } & $1=$ esc. alto riesgo & 1.02 & & 1.17 & & .320 \\
\hline & $2=$ esc. bajo riesgo & .86 & & & & \\
\hline & $3=$ alb. bajo riesgo & .92 & & & & \\
\hline & $4=$ alb. alto riesgo & .88 & & & & \\
\hline
\end{tabular}


Para la variable Locus de control (Andrade, 1984), las diferencias indican mayor locus de control externo en el grupo de AAR y mayor locus de control interno para la escuela EAR (véase tabla 4).

Con relación a la variable Funciones del Yo, se encontró que el grupo AAR tiene mayores Funciones del Yo en el área Dominio competencia respecto a los demás grupos (véase tabla 5).

En el caso de la variable Enfrentamiento como rasgo en los problemas en la vida, se encontraron diferencias estadísticamente significativas que indican mayor enfrentamiento con estilo emocional negativo y evasivo para el grupo de AAR en contraste con los otros gru-

Tabla 4

Análisis de varianza Locus de control

Diferencias por dimensión y grupo

\begin{tabular}{|c|c|c|c|c|c|c|}
\hline Dimensión & Grupos & Media & $\begin{array}{c}\text { Diferencias } \\
\text { entre los grupos }\end{array}$ & $f$ & gl & $\mathbf{P}$ \\
\hline \multirow[t]{4}{*}{ Externo } & $1=$ esc. alto riesgo & 1.38 & $1,3,4$ & 15.98 & 354 & .000 \\
\hline & $2=$ esc. bajo riesgo & 1.30 & 2,4 & & & \\
\hline & $3=$ alb. bajo riesgo & 1.67 & & & & \\
\hline & $4=$ alb. alto riesgo & 1.69 & & & & \\
\hline \multirow[t]{4}{*}{ Interno } & $1=$ esc. alto riesgo & 1.48 & 1,3 & 3.82 & & .010 \\
\hline & $2=$ esc. bajo riesgo & 1.45 & & & & \\
\hline & $3=$ alb. bajo riesgo & 1.35 & & & & \\
\hline & $4=$ alb. alto riesgo & 1.26 & & & & \\
\hline
\end{tabular}

Tabla 5

Análisis de varianza Funciones del Yo

Diferencias por dimensión y grupo

\begin{tabular}{llccccc}
\hline Dimensión & Grupos & Media & $\begin{array}{c}\text { Diferencias } \\
\text { entre los grupos }\end{array}$ & f & gl & P \\
\hline Funciones del Yo & 1 $=$ esc. alto riesgo & 1.74 & & 2.88 & 354 & .036 \\
2 & $=$ esc. bajo riesgo & 1.77 & & & & \\
3 $=$ alb. bajo riesgo & 1.86 & & & \\
4= alb. alto riesgo & 1.92 & & & \\
\hline
\end{tabular}


pos y con la dimensión enfrentamiento directo revalorativo no hubo diferencias (véase tabla 6).

\section{DISCUSIÓN}

En esta investigación se partió de la consideración de que hay diferencias en las características psicológicas en niños por el hecho de vivir en escenarios considerados de riesgo y de no riesgo. Esta hipótesis se confirmó dado que los resultados corroboraron que los niños que viven en albergues por situación de orfandad tienen más baja autoestima, puesto que muestran mayor devaluación social, mayor necesidad por cumplir con expectativas sociales, son inseguros y tienen más aspectos negati- vos de autoestima; asimismo, muestran un locus de control de tipo externo, en comparación con los niños que viven en familia. Lo anterior se relaciona con lo mencionado por Field (1993, en Verduzco, 2004), quien explica que los niños con baja autoestima se sienten aislados, indignos de amor, incapaces de expresarse y defenderse, pasivos, no participativos, susceptibles a la crítica, pesimistas y se sienten controlados por los hechos externos en lugar de sentir que controlan la situación (González Arratia, 2001; Millon, 1998, en Verduzco, 2004). En estos casos, cuando se tiene un bajo concepto de sí mismo, los sucesos estresantes son percibidos con mayor intensidad y las respuestas

Tabla 6

Análisis de varianza Enfrentamiento a los problemas

\begin{tabular}{|c|c|c|c|c|c|c|}
\hline Dimensión & Grupos & Media & $\begin{array}{c}\text { Diferencias } \\
\text { entre los grupos }\end{array}$ & $f$ & gl & $\mathbf{P}$ \\
\hline $\begin{array}{l}\text { Directo } \\
\text { revalorativo }\end{array}$ & $\begin{array}{l}1=\text { esc. alto riesgo } \\
2=\text { esc. bajo riesgo } \\
3=\text { alb. bajo riesgo } \\
4=\text { alb. blto riesgo }\end{array}$ & $\begin{array}{l}4.84 \\
4.66 \\
4.69 \\
5.05\end{array}$ & & 2.52 & 354 & .057 \\
\hline $\begin{array}{l}\text { Emocional } \\
\text { negativo }\end{array}$ & $\begin{array}{l}1=\text { esc. alto riesgo } \\
2=\text { esc. bajo riesgo } \\
3=\text { alb. bajo riesgo } \\
4=\text { alb. alto riesgo }\end{array}$ & $\begin{array}{l}4.34 \\
3.87 \\
4.62 \\
4.85\end{array}$ & 2,4 & 9.14 & & .000 \\
\hline Evasivo & $\begin{array}{l}1=\text { esc. alto riesgo } \\
2=\text { esc. aajo riesgo } \\
3=\text { alb. bajo riesgo } \\
4=\text { alb. alto riesgo }\end{array}$ & $\begin{array}{l}4.43 \\
4.32 \\
4.69 \\
4.78\end{array}$ & & 2.64 & & .049 \\
\hline
\end{tabular}


de enfrentamiento se dan a través de otras personas o con acciones que simplemente descargan sentimientos, pero que no resuelven de ninguna manera el problema, por lo que este se mantiene sin modificar

Por el contrario, los niños que viven en familia muestran un Locus de control interno y más aspectos positivos de la autoestima (familia y escuela). Esto es que el grupo de niños que viven en familia perciben tener el control de las situaciones y una mejor evaluación e imagen de sí mismos. Estos resultados son interesantes en el sentido de que los niños de albergues tienen antecedentes tanto de maltrato como de abandono, además del hecho de ser extraídos de su familia de origen, lo cual denota un fuerte impacto en su autoestima. Asimismo, se ha visto que los niños adoptivos muestran mayor necesidad de atención (Berryman, 1994). Por su parte, Burns (1990) refiere que los niños en desventaja se consideran como posibles víctimas de baja autoestima debido a la discriminación, la pobreza, las expectativas grupales mayoritarias y las condiciones poco estimulantes del medio. Además, se ha observado que los niños huérfanos crecen con esa constante necesidad de ser queridos, de sentirse parte de un ambiente, de ser aceptados por un grupo y de poder expresar sus sentimientos de enojo y agresión; no encuentran la manera de hacerlo, terminando en el refugio de la soledad o en la adhesión a grupos anti- sociales. Desde pequeños viven con el agobio de sentirse desaprobados, buscando cualquier aprobación de las personas que los rodean. Los niños generalmente se muestran temerosos y en alerta para encontrar la aceptación de los maestros y demás compañeros, pero también para detectar cualquier signo de desaprobación y de crítica (Loredo, 1994).

Al hablar de ambas variables (Autoestima y Locus de control), estas diferencias conductuales están relacionadas con la forma en que los individuos enfrentan su medio ambiente, considerándose que los individuos orientados internamente (niños que viven en familia), confían más en sus habilidades y sienten que son capaces de modificar algunas situaciones del medio ambiente; en cambio, los individuos orientados externamente (en este caso niños de albergues), confiarán más en la suerte, el destino o el poder de otros, y, por lo tanto, sentirán que su medio ambiente se debe a fuerzas externas a ellos (Andrade, 1984), lo cual estaría mostrando que el hecho de vivir en familia propicia o es un elemento favorecedor para que los niños desarrollen habilidades para enfrentar los problemas (Fish y Karabenik, 1971, en Andrade, 1984; Dumont y Provost, 1999; Park, 1998).

Entre las diferencias, de acuerdo con la variable Grupo, también fue en la escala de Autoestima, donde se encontró que hay mayor devaluación social, inseguridad y aspectos negativos de la auto- 
estima en los niños de albergue de alto riesgo (AAR); por ello, es indispensable reconocer los efectos negativos de la situación de orfandad en la autoestima de este grupo de niños. En el caso de los niños del albergue de bajo riesgo, ellos cumplen con más características relacionadas con expectativas sociales, como se comentó anteriormente (Field, 1993, en Verduzco, 2004).

Asimismo, en la escala de Locus de control, las diferencias por grupos muestran que los niños de albergue de alto riesgo tienen un locus de control externo, pues creen más que los acontecimientos son producto del destino, la suerte o el azar, mientras que los niños de la escuela de alto riesgo tienen un locus de control de tipo interno. Estas diferencias podrían ser indicadores importantes de la forma como interactúan estos niños en un ambiente significativo.

En la escala de las Funciones del Yo también se encontraron diferencias significativas por grupo. Aquí los niños de albergue de alto riesgo denotan mayor dominio-competencia del Yo; es decir, una mejor adaptabilidad y capacidad de funcionamiento del Yo al ambiente. Sin embargo, esto se tiene que continuar estudiando, dado que solo se aplicó una escala de las doce que componen el instrumento. Esta adaptabilidad del niño, especialmente de albergue, resulta ser un factor positivo, pues, en la medida en que el niño se puede adaptar a diferentes circunstancias, puede desarrollar un mejor equilibrio mental (Bellak, 1994).

Al analizar estadísticamente los datos, con relación al enfrentamiento con problemas en la vida, los niños de albergue, específicamente de alto riesgo, tienden a utilizar un estilo emocional negativo y evasivo, lo cual indica que expresan un sentimiento o una emoción que no resuelve directamente el problema y tienden a evitar o escapar de este. Asimismo, un aspecto interesante y revelador es el predominio del factor emocional negativo que se mantiene en las diferentes situaciones problema (problemas en la vida, familiares, en la escuela, con amigos y de salud). Los resultados obtenidos, por tanto, reflejan esa peculiaridad de estilos de enfrentamiento en la muestra estudiada, donde los individuos expresan algún sentimiento o emoción que no los lleva directamente a la solución del problema, pero pide ayuda a otros, especialmente a los amigos, lo cual es lógico en el caso de los niños de albergues, debido a la ausencia de la familia. Este tipo de enfrentamiento sucede también con los problemas familiares, en la escuela, con los amigos y con la salud, de acuerdo con los resultados que se reportaron en otro estudio (González Arratia, 2007).

Estas conductas se pueden ubicar en el estilo de enfrentamiento dirigido a la emoción, que se caracteriza por las respuestas cognitivas o conductuales, en donde la primera función es manejar 
las consecuencias emocionales del estresor y ayudar a mantener el equilibrio emocional del sujeto (Lazarus y Folian, 1991). Pero también este tipo de estilo de enfrentamiento puede hacer más soportable la vida, evitando las realidades que podrían resultar desbordantes para el sujeto si se enfrentan directamente (Vailant y Pfeiffer, 1977, en Lazarus y Folkman, 1991).

Específicamente en la escala de Relaciones intrafamiliares (Rivera, 1999), se encontraron diferencias significativas por grupo, que indican que en los niños de escuelas de bajo riesgo la media es más alta en la dimensión de expresión, que se refiere a la posibilidad de que los miembros de la familia manifiesten verbalmente sus sentimientos, ideas y experiencias dentro de un clima de respeto.

Lo anterior es un indicador importante de que la familia de estos niños es percibida de una forma adecuada, y confirma la importancia de la familia y particularmente del vínculo de lo que implican el apoyo, la comunicación y el monitoreo como elementos protectores para el niño. En contraste, los niños de albergues de alto riesgo denotan mayores dificultades, que son los aspectos de las relaciones intrafamiliares considerados por el individuo como indeseables, negativos, problemáticos o difíciles.

En este caso, es importante hacer mención de que mientras muchos niños son educados con sus padres, muchos otros son separados de ellos y entregados a orfanatos, en custodia o en adopción a otros hogares, por muerte, divorcio o separación de los padres. Estas son algunas de las formas que pueden dar lugar a las separaciones a largo plazo, y es obvio que esto puede causarles problemas (Berryman, 1994). Por cierto, esta separación implica el cuidado por una persona sustituta. Siguiendo con este autor, el éxito de la crianza por parte de un sustituto va a depender de la edad del niño y de factores como la continuidad del cuidado, su calidad y la actitud social hacia la situación que da lugar al cuidado. El hecho de que los niños de albergue manifiesten mayores dificultades en cuanto a las relaciones intrafamiliares es un indicador importante de que, a pesar de los esfuerzos de las instituciones en la protección de grupos vulnerables (especialmente de niños), requieren de un funcionamiento eficaz para que los cuidados sustitutos sean percibidos de forma positiva. Esto lleva a pensar en la necesidad de implementar programas en torno al desarrollo de habilidades que les permitan a los niños enfrentar las adversidades.

De esta forma, los niños de albergue (de alto y bajo riesgo) presentan baja autoestima, locus de control externo, enfrentamiento a problemas en la vida de tipo emocional negativo y evasivo, dificultad en las relaciones intrafamiliares y mayor adaptación del Yo, lo cual permite suponer que la condición de vida por el hecho de encontrarse en 
un orfanato, es un factor de riesgo que está impactando de manera importante en el funcionamiento psicológico de estos niños, haciéndolos más vulnerables a los factores sociales y más sensibles al desequilibrio entre aspiraciones y posibilidades (Bragagnolo, Rinaudo, Cravero, Martínez \& Vergara, 2006). Esto podría llegar a tener efectos sobre la salud, la capacidad para manejar el estrés, el desarrollo de relaciones interpersonales saludables y la capacidad de proyectar a futuro su crecimiento personal. Por ello, los hallazgos de esta investigación constatan que la familia sigue siendo un factor de protección importante, que permite al niño atenuar el impacto de los factores de riesgo.

Este panorama, que sería preocupante en cualquier grupo, adquiere particular relevancia, pues los resultados nos plantean la necesidad de replicar y profundizar este estudio considerando factores de resiliencia y poniendo énfasis en factores protectores, con el fin de que a pesar de las condiciones adversas en las que se desarrollan los niños, estos no lleguen necesariamente a presentar comportamientos de riesgo.

Finalmente, los resultados pueden contribuir a la creación de modelos de intervención de tal forma que el individuo no solo conozca sus habilidades, sino también que las ponga en ejecución, dado que se consideran indispensables en el desarrollo de la personalidad en contextos de riesgo.

\section{REFERENCIAS}

Andrade, P. P. (1984). "Influencia de los padres en el locus de control de los hijos". Tesis para optar el grado de magíster en psicología social. México: Universidad Nacional Autónoma de México.

Bragagnolo, G.; Rinaudo, A.; Cravero, N.; Martínez, G. \& Vergara, S. (2006). Optimismo, esperanza, autoestima y depresión en estudiantes de Psicología. <http://fpsico. unr.edu.arg/congreso/mesas/Mesa 1 5/bragagnolo_optimismo.pdf $>$.

Bellak, L. \& Goldsmith, L. (1994). Manual para la evaluación de las funciones del Yo (EFY). México: Manual Moderno.

Berryman, J. C. (1994). Psicología del Desarrollo. México: Manual Moderno.

Burns, R. B. (1990). El autoconcepto. Teoría, medición, desarrollo y comportamiento. Bilbao: Ediciones EGA.

Córdova, A. A. (2006). Características de resiliencia en jóvenes usuarios y no usuarios de drogas. Tesis para optar el grado de doctor en psicología. México: Universidad Nacional Autónoma de México.

Donas, D. (1998). Riesgo y vulnerabilidad. Sus posibles aplicaciones en la promoción, prevención, tratamiento y rehabilitación de la salud 
integral de los/las adolescentes. En: Adolescencia al Día, OPS Reproducción de Documentos, V(I), 1-9.

Kotliarenco, M. A., Cáceres, I. \& Fontecilla, M. (1996). Estado del arte en resiliencia. $<$ www.resilienci.cl>. [Consulta: agosto 2003].

Góngora, C. E. \& Reyes, L. I. (2000). El enfrentamiento a los problemas y el locus de control. La psicología social en México, VIII, 165-172.

Góngora, C. E. (2000). "El enfrentamiento a los problemas y el papel del control. Una visión etnopsicológica en un ecosistema con tradición". Tesis para optar el grado de doctor en psicología. México: Universidad Nacional Autónoma de México.

González Arratia, N. I. (2001). Autoestima. Medición y estrategias de intervención, una experiencia en la reconstrucción del ser. México: Universidad Autónoma del Estado de México.

- (2007). Factores determinantes de la resiliencia en niños de la ciudad de Toluca. Tesis para optar el grado de doctor en investigación psicológica. México: Universidad Iberoamericana.

Guadarrama, G. (2001). Condiciones de bienestar y políticas de prevención social en el Estado de México hacia el final del siglo XX. El Colegio Mexiquense 62, 2-17.
Dumont, M. \& Provost, M. A. (1999). Resilience in adolescents: protective role of social support, coning strategies, self-esteem, and Social activities on experiencie on stress and depression. Journal of Youth and Adolescence, 28 (3), 343-363.

Jessor, R. (1993). Successful adolescent development among youth in high-risk settings. The American Psychologist Association, 48 (2), 117-126.

Lazarus, R. S. \& Folkman, S. (1991). Estrés y procesos cognitivos. México: Ediciones Roca.

Loredo, A. A. (1994). Maltrato al menor. México: Mac Graw-Hill.

Martínez, V. P. (2006). Significado psicológico de padre y madre en sujetos albergados en una casa hogar de la ciudad de Toluca. Tesis de licenciatura. México: Universidad Autónoma del Estado de México.

Munist, M.; Suárez, O. E.; Kruskopf, D. \& Silber, T. (2007). Adolescencia y resiliencia. México: Paidós.

Park, C. L. (1998). Stress-related growth and thriving through coping: The roles of personality and cognitive processes. Journal of Social Issues, 54 (2).

Rivera, H. M. E. (1999). Evaluación de las relaciones intrafamiliares: Construcción y validación de una escala. Tesis para optar el grado de magís- 
ter en psicología clínica. México: Universidad Nacional Autónoma de México.

Rojas, M. (2007). Factores de riesgo y protectores identificados en adolescentes consumidores de sustancias psicoactivas. Revisión y análisis del estado actual. [En línea]. $<$ www.cedr.org.pe/ebooks/riesgo $>$ [consulta: 20 de abril del 2007].

Rodríguez, J. (1995). Psicopatología del niño y del adolescente. Sevilla: Universidad de Sevilla.

Rutter, M. (1985). Family and school influences on behavioural development. Journal of Child Psychology and Psychiatry, 22 (3), 349-368.

-. (1987). Psychosocial resilience and protective mechanisms. American
Journal Orthopsychiatry, 57 (3), 316-329.

Sandoval M., M. (2006). La violencia escolar desde la teoría del riesgo y el cambio cultural. Investigaciones del Centro de Estudios en Juventud (CEJU). México: Universidad Católica Silva Henríquez.

Secadas, F. \& Román, J. M. (1984). Psicología evolutiva: edad 10 años. Barcelona: CEAC:

Verduzco, M. A. (2004). Autoestima, estrés y afrontamiento desde el punto de vista del desarrollo. Tesis para optar el grado de doctor. México: Universidad Nacional Autónoma de México. 